Address for Correspondence: Dr. Vinay Mahishale, MD, FCCP, FAPSR, Professor and Head,

Department of Pulmonary Medicine,

J. N. Medical College, KLE University,

Belgaum Karnataka, India.

Email: pulmovinay@yahoo.com

\begin{tabular}{|l|}
\hline Access this article online \\
\hline Website: \\
www.intern-med.com \\
DOI: \\
10.1515/jtim-2015-0019 \\
\hline Quick Response Code: \\
\hline \\
\end{tabular}

\title{
Prevalence and impact of diabetes, hypertension, and cardiovascular diseases in chronic obstructive pulmonary diseases: A hospital-based cross-section study
}

\author{
Vinay Mahishale ${ }^{1}$, Naveen Angadi ${ }^{2}$, Vijayanand Metgudmath ${ }^{3}$, Ajith Eti ${ }^{1}$, \\ Mitchelle Lolly ${ }^{1}$, Sujeer Khan ${ }^{1}$ \\ 'Department of Pulmonary Medicine, J. N. Medical College, KLE University, Belgaum Karnataka, India; \\ 2Department of General Medicine, KLE University, Belgaum Karnataka, India; \\ ${ }^{3}$ Department of Interventional Cardiology, J. N. Medical College, KLE University, Belgaum Karnataka, India
}

\section{ABSTRACT}

Background: Chronic obstructive pulmonary disease (COPD) is associated with important chronic comorbid diseases, including diabetes, hypertension and cardiovascular diseases. As very limited data is available in India, the aim of the present study was to determine the relationship between COPD and the common, chronic comorbid conditions of diabetes mellitus (DM), hypertension (HTN), and cardiovascular diseases (CVD) and also to determine how these affect the clinical course of COPD. Methods: All the COPD cohorts diagnosed as per Global Initiative for Chronic Obstructive Lung Disease-2013 (GOLD-2013) criteria were screened for DM, HTN, and CVD as per stipulated national and WHO guidelines.Results: The prevalence of DM, HTN, and CVD in the 2432 COPD subjects was $25.94 \%, 37.25 \%$, and $13.93 \%$, respectively. In multivariate analyses, very severe COPD was associated with a higher risk of DM (odds ratio [OR] 1.6, 95\% confidence interval [CI] 1.2-2), HTN (OR 1.6, 95\% Cl 1.4-1.9), and CVD (OR 2.5, 95\% Cl 1.9-3.0). Conclusion: A significant relationship was found between COPD and the presence of comorbid DM, HTN, and CVD. It was also found that subjects with advanced COPD were more likely to have at least two of these conditions and hugely affect the outcome of the disease. These findings suggest that the presence of COPD could provide a rationale to look for other comorbid disease and, conversely, that the presence of DM, HTN, or CVD might be the basis for the assessment of patients for airflow limitation and COPD as the tobacco smoking and advancing age were common risk factors.

Key words: COPD, diabetes, hypertension, cardiovascular diseases, comorbidities

\section{INTRODUCTION}

Chronic obstructive pulmonary disease (COPD) is defined by GOLD (Global Initiative for Chronic Obstructive Lung Disease) as a common, preventable, and treatable airflow limitation condition that is usually progressive and is associated with enhanced inflammation in the airways and lungs. ${ }^{[1]}$ COPD is a major cause of morbidity and mortality around the world and projected to become the third leading cause of death worldwide by $2030 \cdot{ }^{[2,3]}$ Although defined by abnormal spirometry, it is well recognized that COPD is more than a respiratory ailment and its impact expands beyond the lungs with significant extrapulmonary consequences. Comorbidities, defined as the co-occurrence of other medical conditions along with COPD, considerably contribute to the severity of disease. Although not essentially associated with the disease, the existence of COPD may actually escalate the risk of other diseases. Comorbidities can occur in patients with 
any degree of airflow limitation and are not restricted to patients with advanced COPD. ${ }^{[4]}$ Furthermore, comorbidities are associated with increased morbidity and mortality and are a major determinant of health status, health expenditure, and prognosis in patients with COPD. ${ }^{[5]}$

Crude estimates suggest that there are 30 million COPD patients in India and contribute significantly to the growing percentage of COPD mortality, which is estimated to be among the highest in the world; that is more than 64.7 estimated age standardized death rate per 1,00,000 in both sexes. This would translate to about 556,000 cases in India $(>20 \%)$ out of a world total of $2,748,000$ annually. Such enormous volumes of disease have the potential to have devastating impact on the health systems and state economies. ${ }^{[6,7]}$ In India, there is also escalating epidemic of chronic noncommunicable diseases (NCDs) like diabetes mellitus (DM), hypertension (HTN), and cardiovascular diseases (CVD) ${ }^{[8]}$ If mortality due to comorbid conditions like DM, HTN, and CVD associated with COPD are taken together, then the convergence of these noncommunicable chronic diseases pose a great impact on the severity and outcome of the disease.

The presence of these common comorbidities is substantially affecting the outcome of COPD at various levels of healthcare services. As very limited data is available, the exact prevalence of these comorbidities in COPD patients in India is not known. The aim of the present study was to determine the relationship between COPD and the common, chronic comorbid conditions of DM, HTN, and CVD and also to determine how these affect the clinical course of COPD.

\section{MATERIALS AND METHODS}

\section{Source of Data}

Inpatient and outpatient Departments of Pulmonary Medicine, Internal Medicine, Endocrinology and Cardiology Departments at a tertiary care hospital at Belgaum district of Karnataka in India.

\section{Population}

All subjects aged more than 40 years and diagnosed as cases of COPD as per GOLD criteria. ${ }^{1}$

\section{Study Design}

A cross-section study.

\section{Study Period}

Primary data was collected between January 1, 2013 and December 31, 2014.

\section{Inclusion Criteria}

(1) Both male and female patients diagnosed with COPD.

(2) Age $>40$ years.

\section{Exclusion Criteria}

Patients were excluded from the study if they have any of the conditions like:

1. Any chronic lung disease other than COPD

2. HIV infection

3. Connective tissue disorders

4. Chronic renal failure

5. Chronic liver disease

6. Malignancies on long-term steroid or cytotoxic drug therapy

7. Chronic alcoholics.

Baseline data was recorded which included age, sex, biomass fuel exposure, symptoms related to the respiratory system, DM, HTN, and CVD with duration of illness, level of dyspnea (Medical Research Council range [0-4]), smoking status (current or nonsmoker or ex-smoker), pack years, current treatment, previous medications, occupation, and number of exacerbations, that is, emergency hospital admissions or unscheduled hospital visits in the last 1 year. Also dyspnea score (D), level of airflow obstruction (O), current smoking status (S), and exacerbations (E) (DOSE) score were noted. Mortality has been found to be associated with patients with a DOSE index score $>4{ }^{\left[{ }^{[9]}\right.}$

\section{COPD Diagnosis}

All the participants were subjected to spirometry and patients with post bronchodilator FEV1/FVC $<70 \%$ predicted were considered as cases of COPD. Then they were categorized as mild, moderate, severe, and very severe COPD patients as per the GOLD guidelines ${ }^{[1]}$

\section{DM, HTN, CVD Screening}

All the subjects enrolled in the study are screened for DM, HTN, and CVD as per stipulated national guidelines with cutoff thresholds in line with those recommended by WHO. ${ }^{[8]}$

In brief, fasting blood sugar (FBS) $>126$ indicates DM and $\mathrm{FBS}<110 \mathrm{mg} / \mathrm{dL}$ is normal. Patients whose diabetes status was uncertain underwent random blood sugar (RBS) testing, and if was more than $126 \mathrm{mg} / \mathrm{dL}$, the subjects were further assessed with FBS and postprandial blood sugar (PPBS). If FBS was more than $126 \mathrm{mg} / \mathrm{dL}$ or PPBS more than $200 \mathrm{mg} / \mathrm{dL}$ the subjects were confirmed as having $\mathrm{DM}$ at the baseline.

Subjects were classified as having HTN if they reported physician diagnosis of HTN, were receiving treatment for HTN, or had evidence of same upon examination (diastolic blood pressure $90 \mathrm{mmHg}$ or a systolic blood pressure 140 $\mathrm{mmHg}$, based on three measurements). 
Subjects reporting a diagnosis of a prior myocardial infarction, stroke, heart failure, angina, or transient ischemic attacks were classified as having cardiovascular disease at the baseline examination after panel discussion.

\section{Panel Discussion}

All participants were evaluated by a consensus panel that decided after plenary discussion whether a diagnosis of DM, HTN, or CVD was present, possible, or absent. The panel was consisted of pulmonologist, general physician, and a cardiologist. Diagnosis of COPD was confirmed as per the guidelines of the GOLD program, which provides standard diagnostic criteria, severity staging as well as recommendations for prevention and management of COPD. The panel confirmed diagnosis of DM, HTN, and CVD if the subjects met the WHO criteria for these disorders. Characteristics of the participants in the screening were tabulated against the COPD status defined as no comorbidity "COPD +0 ," COPD with any one of three comorbidities "COPD +1 ," COPD with any two of three conditions "COPD +2 ," and finally COPD with all three conditions as "COPD +3 ."

\section{RESULTS}

The final cohorts consisted of 2432 COPD patients with $1648(67.76 \%)$ males and 784 females (32.24\%). About 1351 $(55.55 \%)$ were smokers whereas $653(26.85 \%)$ had history of biomass fuel exposure (Table 1). Analyses showed that increasing age, a higher BMI, lower education status, longer duration of COPD, higher pack years of smoking, high DOSE score and male sex were associated with a higher risk of DM, HTN, and CVD (Table 2). In multivariate analyses, very severe COPD was associated with a higher risk of DM (odds ratio [OR] 1.6, 95\% confidence interval [CI 1.2-2), HTN (OR 1.6, 95\% CI 1.4-1.9), and CVD (OR 2.5, 95\% CI 1.9-3.0). Similar findings were seen for other stages of COPD (Table 3).

In the present study cohort, $912(37.5 \%)$ subjects had no comorbid disease, $852(35.03 \%)$ had one comorbid disease, $461(18.5 \%)$ had two comorbid diseases, and 207 (8.5\%) had three comorbid diseases (Table 1). Multinomial logistic regression showed that compared to subjects with normal lung function, those with very severe COPD were more likely to have one (OR 1.8, 95\% CI 1.4-2.3), two (OR

Table 1: Patients included in this study.

\begin{tabular}{lll}
\hline & $N$ & $\%$ \\
\hline COPD subjects in the study & 2432 & 67.76 \\
\hline Males & 1648 & 32.24 \\
Females & 784 & 55.55 \\
Tobacco smokers & 1351 & 26.85 \\
Subjects with biomass exposure & 653 & 37.5 \\
Patients with "O" comorbidity- “COPD + 0" & 912 & 35.03 \\
Patients with "1" comorbidity- “COPD + 1" & 852 & 18.95 \\
Patients with "2" comorbidities-“COPD +2" & 461 & 8.5 \\
Patients with "3" comorbidities-“COPD + 3" & 207 & 25.94 \\
Subjects with DM & 631 & 37.25 \\
Subjects with HTN & 906 & 13.93 \\
Subjects with CVD & 339 & \\
\hline
\end{tabular}

Table 2: Baseline demographic characteristics of all subjects.

\begin{tabular}{|c|c|c|c|c|}
\hline DATA & COPD + 0 & COPD+1 & COPD +2 & COPD+3 \\
\hline Number & 912 & 852 & 461 & 207 \\
\hline Age & $54 \pm 8.2$ & $59 \pm 9.13$ & $63 \pm 7.3$ & $67 \pm 10.28$ \\
\hline BMI & $18.34 \pm 2.48$ & $19.23 \pm 3.63$ & $22 \pm 4.21$ & $22 \pm 4.68$ \\
\hline Smoking (pack years) & $6.34 \pm 3.9$ & $7.21 \pm 2.3$ & $8.16 \pm 3.2$ & $8.20 \pm 2.28$ \\
\hline Duration of COPD (years) & $6.83 \pm 2.8$ & $8.21 \pm 2.3$ & $9.42 \pm 3.9$ & $10.41 \pm 8.93$ \\
\hline Mean FEV $1 \%$ predicted & $68.38 \pm 13.8$ & $63.2 \pm 12.1$ & $57.14 \pm 8.9$ & $51 \pm 13.26$ \\
\hline Dose score & $2.18 \pm 2.3$ & $2.96 \pm 1.06$ & $4.96 \pm 1.04$ & $5.56 \pm 1.96$ \\
\hline
\end{tabular}


Mahishale et al.: Prevalence and impact of diabetes, hypertension and cardiovascular diseases in COPD

\begin{tabular}{|c|c|c|c|}
\hline GOLD Category & DM & HTN & CVD \\
\hline Very severe & $1.6(1.2-2)$ & $1.6(1.4-1.9)$ & $2.5(1.9-3)$ \\
\hline Severe & $1.5(1.2-1.7)$ & $1.4(1.3-1.5)$ & $2.4(1.9-2)$ \\
\hline Moderate & $0.9(0.7-1.2)$ & $1.3(1.2-1.4)$ & $2.5(1.5-2.6)$ \\
\hline Mild & $1.2(1.1-1.4)$ & $1.2(1.1-1.3)$ & $2.4(2.1-2.5)$ \\
\hline \multirow[t]{2}{*}{ Normal } & 1 & 1 & 1 \\
\hline & Comorbiditie & & \\
\hline Gold Category & 1 & 2 & 3 \\
\hline Very severe & $1.8(1.4-2.3)$ & $3.6(2.6-3.4)$ & $4(3.6-4.9)$ \\
\hline Severe & $1.5(1.3-1.6)$ & $3.2(2.4-4)$ & $3.9(2.9-4.9)$ \\
\hline Moderate & $1.4(1.4-1.7)$ & $3.2(2.6-3.6)$ & $3.8(2.8-4.6)$ \\
\hline Mild & $1.4(1.4-1.6)$ & $3(2.6-3.5)$ & $3.6(2.8-4.2)$ \\
\hline Normal & 1.0 & 1.0 & 1.0 \\
\hline
\end{tabular}

3.6, 95\% CI 2.6-3.4), or three (OR 4, 95\% CI 3.0-4.1) comorbid diseases, with similar observations in other groups too (Table 4).

\section{DISCUSSION}

NCDs, including COPD, DM, HTN, and CVD are the major global health problem of the century. ${ }^{[10]}$ They are the world leading cause of disease burden and mortality and are increasing in prevalence even in low- and middleincome countries like India. The costs incurred by the convergence of these uncontrolled diseases are substantial with momentous impact on each others' outcome. ${ }^{[1]}$ The association is bidirectional as COPD is known to escalate the prevalence and severity of these NCDs and vice versa. COPD is the major respiratory NCD in our country along with DM and HTN. As far as our knowledge extends, this is the first kind of study in which association between COPD and these NCDs was assessed.

The understanding of COPD has changed considerably over the past two decades. The definition of the disease has moved from a simple airflow limitation (forced expiratory volume in $1 \mathrm{~s}, \mathrm{FEV}_{1}$ )-centric view of the disease to the understanding that COPD as a complex and heterogeneous condition. It is important to highlight that, "complex" means that COPD has a number of intrapulmonary and extrapulmonary components whose dynamic interactions along time are not linear, whereas "heterogeneous" ${ }^{[12]}$ indicates that not all of these components are present in all individuals at any given time point. The presence of multiple comorbidities adds to the complexity and heterogeneity of
COPD. The universal aging of the world's population is reinforcing this trend, partly due to the fact that the prevalence is higher in age groups. Epidemiological studies and large clinical trials have helped us to understand the importance of comorbidities. ${ }^{[13-15]}$ Greater understanding of the pathophysiology of COPD, focused on the concept of systemic inflammation, has also helped to explain the high frequency of major comorbidities (cardiovascular, DM, HTN) in addition to coexisting illnesses that one would naturally expect due to the patients' advanced age and due to the shared risk factors such as tobacco smoking, sedentary life style, etc.

The present study revealed that prevalence of DM in study cohorts was $25.94 \%$, which is considerably higher than the national average of $8 \% \cdot{ }^{[16,17]}$ COPD is known to be novel risk factor for development of DM via multiple pathophysiological modifications such as inflammation, oxidative stress, insulin resistance, weight gain and alterations in metabolism of adipokines. ${ }^{[18]}$ In the present study, the risk of DM increased with the severity of COPD (odds ratio $[\mathrm{OR}] 1.6,95 \%$ confidence interval [C] 1.2-2 in very severe $\mathrm{COPD}$ ). Instabilities in glucose metabolism are more frequent in COPD patients than in non-COPD individuals. Also, almost half of all COPD patients suffer from other medical problems frequently linked to diabetes, such as HTN and higher levels of cholesterol. The combination of these medical problems is sometimes referred to as "metabolic syndrome" and is considered a cause for various cardiovascular complications in COPD patients. ${ }^{[19]}$ Similarly, many studies have found that DM causes an accelerated decline in lung functions as compared to nondiabetics. 
We also demonstrated that the risk of HTN and CVD is significantly high in COPD subjects compared to general population. We noted that the risk of HTN and CVD also increased significantly with severity of COPD. In an analysis of worldwide data for the global burden of HTN, $20.6 \%$ of Indian men and $20.9 \%$ of Indian women were suffering from HTN in 2005. The rates for HTN in percentage are projected to go up to 22.9 and 23.6 for Indian men and women, respectively by $2025 .{ }^{[20]}$ However, in present analysis prevalence of HTN in COPD patients is much more higher than this projected data. HTN exerts a substantial public health burden on COPD subjects and severely affects quality of life of these subjects. Also exerts greater risk of CVD. It is interesting to note that of a total of 9.4 million deaths in India in 1990, CVD caused 2.3 million deaths (25\%). A total of 1.2 million deaths were due to coronary heart disease and 0.5 million due to stroke. It has been projected that by 2020 , there would be a $111 \%$ increase in CVD deaths in India. This increase is much more than $77 \%$ for China, $106 \%$ for other Asian countries, and 15\% for economically developed countries. ${ }^{[21]}$ Such an alarming escalation of epidemics of HTN and CVD will be further augmented by the presence of COPD itself.

Our observations that increasing age, a higher BMI, lower education status, longer duration of COPD, higher pack years of smoking, high DOSE score, and male sex were associated with a higher risk of DM, HTN, and CVD in present cohorts is in agreement with the study by Mannino et al. ${ }^{[2]}$ We also established that increase in number of comorbidities in COPD leads to increase in the severity of the disease, high symptom score, exacerbations, frequent emergency visits, limited physical activity, and poor quality of life as per DOSE score data. In the current study number of tobacco smokers was notably high. Tobacco exposure is common risk factor for both COPD and the comorbidities discussed here. There are about 120 million smokers in India, which is corresponding to $12 \%$ of the world's smokers. Approximately 900,000 people die every year in India due to smoking as of 2009. According to 2002 WHO estimate, $30 \%$ of adult males in India smoke. Among adult females, the figure is much lower at between 3 and $5 \%$. Tobacco is usually consumed in the form of bidis in rural areas, which are smaller than cigarettes and contain only about a quarter as much raw tobacco, wrapped in the leaf of another plant. ${ }^{[23]}$

Smoking is projected to delay the millennium development goals of WHO. The present study as well as many studies discussed above emphasizes the urgent need for smoking cessation programs at all levels of healthcare services. It is essential to note that interventions to decrease the prevalence of smoking among patients with COPD (or in the general population) may have an important impact on the incidence of COPD and comorbidities. The interventions including counseling and pharmacological treatment for cessation of smoking in COPD patients are still far from being implemented in routine clinical practice, particularly in countries like India where high proportions of COPD patients continue to smoke and come from remote rural dwellings.

High proportion of subjects in the current study was older than 60 years and we observed advancing age was significant risk for multimorbidity in COPD subjects. Mean age of "COPD +3 " subjects was $67 \pm 10.28$, which was remarkably more than other groups in the study. It is worth mentioning here that the world is experiencing the dramatic change in the demographics of the population with the strata of older individuals growing faster than the younger individuals due to decreasing population growth rate and increased life expectancies. United Nations Population Division reported that the global share of older people (aged $\geq 60$ years) increased from $8 \%$ in 1950 and $9 \%$ in 1990 to $12 \%$ in 2013, and will continue to grow to an estimated $21 \%$ by 2050 . By 2045-2050, life expectancy is projected to reach 83 years in the more developed regions of the world and 75 years in the less developed regions of the world. There is another important noteworthy epidemiological transition, which is the movement from communicable diseases toward NCD, through the world. ${ }^{[23,24]}$

COPD is one of the important NCD in the world and accelerated prevalence of other NCD like DM, HTN, and CVD will have profound negative impact on disease outcome. Hence it is imperative to screen all COPD patients for DM, HTN, and CVD. The increased understanding of COPD as a systemic disease has significantly changed the understanding of pathophysiology of comorbidities. In addition to conventional pharmacological therapy focused on treating chronic airflow limitation, management of COPD now requires a more holistic approach, including the diagnosis and appropriate treatment for comorbid conditions like DM, HTN, and CVD.

\section{Strengths of the study are-}

1. Large sample size.

2. It focuses on a specific group of subjects (COPD) at high risk of DM, HTN, and CVD: those who are symptomatic and elderly.

3. The screening was based not only on the symptomatology but also on a detailed individual assessment by a multidisciplinary panel (pulmonologist, a general physician, and a cardiologist) that took into account clinical history, 
risk factors, physical examination, blood tests, and all other relevant investigations to arrive at the diagnosis of comorbidities.

Limitations: The study is limited by the fact that it is a single centric cross-section study and hence the outcomes of the trial cannot be generalized. COPD exhibits many other comorbidity like skeletal muscle dysfunction, depression, etc., that were not involved in the study might as well contribute to the outcome. A further study with involvement of different geographic populations with wide range of comorbidities is worth exploring.

\section{CONCLUSION}

In conclusion, a significant relationship was found between COPD and the presence of comorbid DM, HTN, and CVD. It was also found that subjects with advanced COPD were more likely to have at least two of these conditions and hugely affect the outcome of the disease. These findings suggest that the presence of COPD could provide a rationale to look for other comorbid disease and, conversely, that the presence of DM, HTN, or CVD might be the basis for the assessment of patients for airflow limitation and COPD as the tobacco smoking and advancing age were common risk factors.

\section{Conflicts of Interest}

\section{None declared.}

\section{REFERENCES}

1. Global strategy for the diagnosis, management, and prevention of chronic obstructive pulmonary disease. Global Initiative for Chronic Obstructive Lung Disease website. Available at: http://www.goldcopd. org/guidelines-global-strategy-for-diagnosis- management.html. Accessed on August 26, 2015.

2. Mathers CD, Loncar D. Projections of global mortality and burden of disease from 2002 to 2030. PLoS Med 2006; 3: e442.

3. Mahishale V, Mahishale A, Patil B, Sindhuri A, Eti A. Screening for diabetes mellitus in patients with chronic obstructive pulmonary disease in tertiary care hospital in India. Niger Med J 2015;56:122-5.

4. Agusti A, Calverley PM, Celli B, Coxson HO, Edwards LD, Lomas DA, et al. Characterisation of COPD heterogeneity in the ECLIPSE cohort. Respir Res 2010;11:122.

5. Mannino DM, Thorn D, Swensen A, Holguin F. Prevalence and outcomes of diabetes, hypertension and cardio-vascular disease in COPD. Eur Respir J 2008; 32: 962-9.

6. Salvi S, Agarwal A. India needs a national COPD prevention and control program. J Assoc Physicians India 2012; 60: S5-7.

7. Mahishale V, Mahishale A, Patil B, Eti A, Lolly M, Khan S. Screening for chronic obstructive pulmonary disease in elderly subjects with dyspnoea and/or reduced exercise tolerance - A hospital based cross sectional study. Egypt J Chest Dis Tuberculosis 2015: 64: 567-71.
8. Directorate General of Health Services, India. National programme for prevention and control of cancer, diabetes, cardiovascular disease and stroke (NPCDCS). Available from: http://health.bih.nic.in/Docs/ Guidelines-NPCDCS.pdf (Accessed on June 12, 2015).

9. Jones RC, Donaldson GC, Chavannes NH, Kida K, Dickson-Spillmann M, Harding S, et al. Derivation and validation of a composite index of severity in chronic obstructive pulmonary disease: The DOSE Index. Am J Respir Crit Care Med 2009;180:1189-95.

10. Rosenbaum L, Lamas D. Facing a "Slow-Motion Disaster" - The UN Meeting on Noncommunicable Diseases. New Engl J Med 2011; 365:2345-8.

11. Mannino DM, Buist AS. Global burden of COPD: risk factors, prevalence, and future trends. Lancet 2007; 370:765-73.

12. Agusti A. Thorax 2014; 69:857-864.

13. Crisafulli E, Costi S, Luppi F, Cirelli G, Cilione C, Coletti O, et al. Role of comorbidities in a cohort of patients with COPD undergoing pulmonary rehabilitation. Thorax 2008; 63: 487-92.

14. Celli BR, Thomas NE, Anderson JA, Ferguson GT, Jenkins CR, Jones PW, et al. Effect of pharmacotherapy on rate of decline of lung function in chronic obstructive pulmonary disease: Results from the TORCH study. Am J Respir Crit Care Med 2008;178:332-8.

15. Mahishale V. Ageing world: Health care challenges. J Sci Society 2015; 42: $138-43$.

16. India Tuberculosis-Diabetes Study Group. Screening of patients with tuberculosis for diabetes mellitus in India. Trop Med Int Health 2013;18:636-45.

17. Jali MV, Mahishale VK, Hiremath MB. Bidirectional screening of tuberculosis patients for diabetes mellitus and diabetes patients for tuberculosis. Diabetes Metab J 2013;37:291-5.

18. Rimm EB, Manson JE, Stampfer MJ, Colditz GA, Willett WC, Rosner B, et al. Cigarette smoking and the risk of diabetes in women. Am J Public Health 1993;83:211-4.

19. Cavaillès A, Brinchault-Rabin G, Dixmier A, Goupil F, Gut- Gobert C, Marchand-Adam S, et al. Comorbidities of COPD. Eur Respir Rev 2013;22:454-75.

20. Kearney PM, Whelton M, Reynolds K, Muntner P, Whelton PK, He J. Global burden of hypertension: analysis of worldwide data. Lancet 2005; 365:217-23.

21. Rodgers A, Lawes C, MacMahon S. Reducing the global burden of blood pressure related cardiovascular disease. J Hypertens 2000; 18(Suppl 1): S3-S6.

22. Mannino D, Swensenand Holguin F. Prevalence and outcomes of diabetes, hypertension and cardiovascular disease in COPD. Eur Respir J 2008; 32: 962-9.

23. Mahishale V, Patil B, Lolly M, Eti A, Khan S. Prevalence of Smoking and Its Impact on Treatment Outcomes in Newly Diagnosed Pulmonary Tuberculosis Patients: A Hospital-Based Prospective Study. Chonnam Med J 2015;51:86-90.

24. United Nations. World Population Ageing 2013. Available at: www. un.org/ en/development/desa/population/publications/pdf/ageing/ WorldPopulationAgeing Report 2013.pdf. Accessed on December 27, 2014.

25. Lozano R, Naghavi M, Foreman K, Lim S, Shibuya K, Aboyans V, et al. Global and regional mortality from 235 causes of death for 20 age groups in 1990 and 2010: a systematic analysis for the Global Burden of Disease Study 2010. Lancet 2012; 380: 2095-128.

How to cite this article: Mahishale V, Angadi N, Metgudmath V, Eti A, Lolly M, Khan S. Prevalence and impact of diabetes, hypertension, and cardiovascular diseases in chronic obstructive pulmonary diseases: A hospital-based cross-section study. J Transl Intern Med 2015; 155-160. 